\title{
SCUBA Observations of the Host Galaxies of Gamma-Ray Bursts
}

\author{
V. E. Barnard*, N. R. Tanvir ${ }^{\dagger}$, A. W. Blain**, A. Fruchter ${ }^{\ddagger}$, C. \\ Kouveliotou $^{\S}$, P. Natarajan ${ }^{\mathbb{I}}$, E. Ramirez-Ruiz, E. Rol ${ }^{\dagger \dagger}$, I. A. Smith ${ }^{\dagger}$, R. \\ P. J. Tilanus* and R. A. M. J. Wijers ${ }^{\dagger \dagger}$ \\ ${ }^{*}$ Joint Astronomy Centre, Hilo, USA \\ ${ }^{\dagger}$ University of Hertfordshire, Hatfield, UK \\ ${ }^{* *}$ Caltech, Pasadena, USA \\ ${ }^{\sharp} S T S I$, Baltimore, USA \\ ${ }^{\S}$ MSFC, Huntsville, USA \\ "Yale University, New Haven, USA \\ "IoA, Cambridge, UK \\ ${ }^{\dagger}$ University of Amsterdam, Netherlands \\ ${ }^{\sharp}$ Rice University, Houston, USA
}

\begin{abstract}
In recent years, a population of galaxies with huge infrared luminosities and dust masses has been discovered in the submillimetre. Observations suggest that the AGN contribution to the luminosities of these submillimetre-selected galaxies is low; instead their luminosities are thought to be mainly due to strong episodes of star formation following merger events. Our current understanding of GRBs as the endpoints in the life of massive stars suggest that they will be located in such galaxies.

We have observed a sample of well-located GRB host galaxies in the submillimetre. Comparing the results with the general submillimetre-selected galaxy population, we find that at low fluxes $\left(S_{850} \leq 4 \mathrm{mJy}\right)$, the two agree well. However, there is a lack of bright GRB hosts in the submillimetre. This finding is reinforced when the results of other groups are included. Possible explanations are discussed. These results help us assess the roles of both GRB host galaxies and submillimetreselected galaxies in the evolution of the Universe.
\end{abstract}

\section{STAR FORMATION IN THE SUBMILLIMETRE}

Our understanding of galaxy evolution was revolutionised by the observations of the IRAS satellite [1], which revealed large populations of dust-enshrouded, actively starforming galaxies in the local Universe. This picture was extended to high redshifts by several instruments, predominantly the Submillimetre Common-User Bolometer Array (SCUBA, [2]) on the James Clerk Maxwell Telescope (JCMT). SCUBA observes simultaneously at 450 and $850 \mu \mathrm{m}$ and hence is sensitive to emission from dust, heated to $40 \mathrm{~K}$ by optical and UV light form stars. SCUBA's unique sensitivity to submillimetre (submm) radiation is enhanced by the negative k-correction suffered by the greybody radiation, which makes SCUBA almost equally able to see galaxies at $z$ of 1 and 10 .

SCUBA has now discovered in excess of 200 galaxies; however progress in understanding these objects has been relatively slow. Identification of the SCUBA galaxies at optical wavelengths is hampered by two factors. Firstly, SCUBA galaxies are inherently 
faint in the optical (typically $I>26$ [3]). Secondly, at SCUBA's primary wavelength of $850 \mu \mathrm{m}$, the angular resolution is only $\sim 14$ ". The surface density of suitably optically faint galaxies is too high to then allow secure identifications. An alternative, but extremely time-consuming route, is to make interferometric detections of $\mathrm{CO}$ molecular lines in the millimetre $[4,5,6]$, providing a position at which to search with optical telescopes. Only five redshifts have thus been obtained so far for SCUBA-selected galaxies.

However recent studies by Chapman et al. [7] have provided some progress, using a pre-selection in the radio to obtain redshifts for several tens of SCUBA galaxies. This technique has shown that SCUBA galaxies are at $z=0.7-3.7$, with a median of 2.4. Furthermore, optical observations of these radio-selected SCUBA galaxies reveal that they are large systems, with irregular morphologies suggesting that they are in the earliest stages of mergers with enhanced star formation as a result [8]. These results confirm that much, perhaps the majority, of high-redshift star formation takes place in massive dusty systems with luminosities $L>10^{12} \mathrm{~L}_{\odot}$ [9].

Inclusion of the SCUBA galaxy population is thus essential for any study of the star formation history of the Universe, but with SCUBA's confusion limit at around $2 \mathrm{mJy}$, the population seen by SCUBA accounts for only about $30 \%$ of the submm background detected by $C O B E$-FIRAS. Furthermore the role of AGN in the huge luminosities of SCUBA galaxies is unclear; a recent estimate suggests hard X-ray AGN are found in only $\sim 10 \%$ of SCUBA galaxies [10] but the evolution of SCUBA galaxies is not yet understood; several studies suggest that they are the high-redshift precursors of the local Universe massive spheroids $[11,12,13]$ since their star formation rates and volume density match that predicted by evolutionary scenarios. To make progress in understanding both SCUBA galaxies and their role in the history of the Universe, we must look to other observations.

\section{GRBS AS TRACERS OF STAR FORMATION}

Several recent pieces of evidence link GRB events with star forming regions in their host galaxies. The recent coeval detection of supernova SN2003dh with GRB 030329 $[14,15]$ in particular has firmly established the association between GRBs and massive stars. Since high-mass stars are short-lived, the regions of galaxies surrounding GRBs are likely to be associated with ongoing star formation. Hence GRBs may be an alternative way to measure star formation in the Universe. They have several advantages in this regard. Their extreme luminosity means that they can be seen, if they exist, at extremely high redshifts, even through high intervening column densities. This ties in well with SCUBA since as explained above, SCUBA has a similar redshift-independent sensitivity. GRBs can pinpoint star formation in previously undetected objects; and hence if GRB rates can be shown to correlate with star formation it will not even be necessary to observe the host galaxy to include its contribution to the history of the Universe. Furthermore a great deal of other information can be obtained from the afterglow observations alone, such as metallicities, column densities etc, and this technique will not suffer from the impact of AGN.

However GRBs suffer from certain disadvantages too. GRBs can suffer from large 
selection effects; in order to know more about a GRB it is essential that its afterglow be discovered. This biases studies to those GRBs which take place in a sufficiently dense ISM for the afterglow to be detected, as well as to low- $z$ and low-dust environments, although the GRB will clear out the dust in its very local environment [16]. We do not yet know whether the variety seen in GRBs is due to the progenitors or the ISM environments, but the effect of varying observing strategies must also be considered.

\section{OBSERVATIONS OF GRB HOST GALAXIES WITH SCUBA}

To make progress in understanding the relationship that both GRBs and SCUBA galaxies have with global star formation, we have undertaken two studies to observe GRB host galaxies with SCUBA. In our first study [17], we observed a small sample of so-called 'dark bursts', GRBs for which optical afterglows were not found. A simple explanation of these events may be that dust obscures the optical afterglow, which would make the host galaxies prime candidates for SCUBA galaxies. Recently the afterglow of GRB 030115 (see article in this proceedings) was detected in the infrared but not the optical, demonstrating that some dark bursts may be due to dust obscuration. We observed the hosts of five GRBs ${ }^{1}$ whose afterglows were seen at either radio or X-ray wavelengths, but not at optical wavelengths despite deep, rapid searches.

Of this sample, only one source, GRB 000210, gave a marginal detection, which was confirmed when combined with further data by Berger et al. [19]. This rate of detection matches that predicted by a model which assumes that SCUBA galaxy incidence and GRB rates are both directly correlated with the global star formation rate [20]. Whilst this was only a small sample, the result supports the idea that GRBs and SCUBA galaxies provide complementary measures of star formation in the Universe. However it also indicates that 'dark bursts' are not always reliable signposts to dusty host galaxies; classifying all dark bursts as one type of object is unlikely to be physically accurate.

In our second study (Tanvir et al. in prep.) we observed all remaining accurate GRB locations with SCUBA to measure whether the apparent relation between SCUBA galaxies and GRB hosts continued with a larger unbiased sample. Combining our results with those of a similar study by Berger et al. [19], we have a sample of 20 hosts with $<1.4 \mathrm{mJy}$ errors. The results are plotted in Fig. 1. The histogram represents the binned observational results, whereas the data points represent the predictions of the model by Ramirez-Ruiz et al. ([20]) mentioned above. The error bars indicate the counting statistics in each observational bin.

This figure indicates that the observations agree reasonably well with the model. However, the observational results are skewed to lower fluxes than the model predicts. Of $20 \mathrm{GRBs}$, only three hosts were detected, and the maximum flux was $3.74 \mathrm{mJy}$ for GRB 010222 [21]. No host was found to have a flux above $4 \mathrm{mJy}$, whereas the model predicts that $>10 \%$ of GRB hosts should be in this bin. Whilst the statistics are not

\footnotetext{
${ }^{1}$ Note that in Barnard et al. [17], the observations of GRB 001025 were thought to have used an unreliable position. However later analysis [18] suggests that the observed position was the correct position of the GRB after all, and hence the results for this GRB are included here.
} 


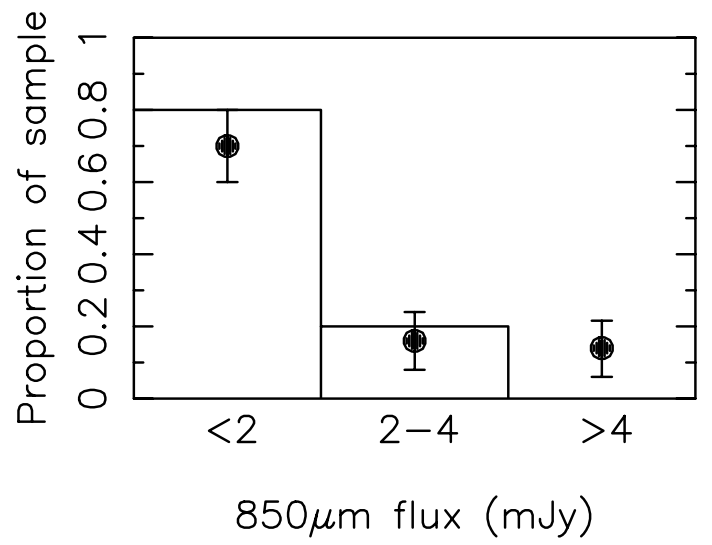

FIGURE 1. Results of the wide sample of all well-located GRB locations with SCUBA. Histogram is observational results binned into three (different-width) bins; points with error bars show prediction of a model by Ramirez-Ruiz et al. ([20]) convolved with observational errors and adjusted for confusion effects. The error bars represent the counting statistics of the proportion expected in each bin.

yet strong enough to confirm this apparent departure from the model, it is interesting to speculate on possible causes.

Firstly, we note that there is likely to be a small population of truly dark bursts - such as GRB 030115 - where dust obscures the site of the burst so that its afterglow is not discovered. Further infrared and submm observations of GRB afterglows are necessary to quantify this population. A similar selection effect may be towards detecting GRBs preferentially at low redshifts - whilst the GRBs themselves can be detected at high redshifts, the afterglow observations are limited. Since the recent work of Chapman et al. ([7]) confirms that SCUBA galaxies are a high-redshift population this may cause an important bias.

Alternatively, it may be the case that the submm-luminous phase and the GRB epoch of the host galaxies do not coincide. Chapman et al. ([8]) used their recent HST observations of the radio-selected sample to model the submm evolution of the SCUBA galaxies; for one example they concluded that the submm-luminous phase may be as brief as 5 Myr. Hence the GRB events may occur in these galaxies after the submm flux has dwindled, and we only see in the submm those few whose flux has a slower decline.

Another explanation may be that GRBs occur preferentially in low-metallicity systems, which is predicted as a consequence of the popular collapsar model [22]. Whilst low metallicities have been inferred for several GRB hosts (for example [23]) the fact that three submm hosts have been confirmed by our studies and those of Berger et al. [19] demonstrates that there is not a complete anti-correlation. It is likely in any case that starbursting galaxies have a very patchy distribution of dust and gas.

Another issue is the extent to which SCUBA galaxy fluxes are boosted by AGN dust heating. If AGN in fact have a larger impact on SCUBA galaxy fluxes then the model points in Fig. 1 are inappropriate. If SCUBA galaxies are, as is thought, proto-elliptical galaxies, then all models predict a QSO phase at some point after the onset of the merger 
event $[11,12,13]$. However the impact of the QSO on the submm flux, and its epoch of activity relative to star formation, has not been strongly quantified yet.

Finally we note that the three detected submm hosts show some differences from the general (submm brighter) SCUBA galaxy population. Whilst SCUBA galaxies show a surprising variety in the optical [3], the GRB hosts seem to be bluer and perhaps more compact [19]. They are also found at lower redshifts than the general SCUBA galaxy population, all being at $z<1$. However this is for only a very small sample which, as noted above, may already be biased towards low redshifts.

\section{CONCLUSIONS}

We have completed an initial survey of the hosts galaxies of GRBs in the submm, in an attempt to understand how both populations relate to star formation rates in the Universe as a whole. We find that whilst there is a general overlap between the host population and the known SCUBA galaxy population, there is a dearth of bright GRB hosts in the submm. Suggestions to explain this disparity include both sample selection effects and physical factors such as timescales of the various phases of galaxy luminosity, metallicity in GRB hosts and the unknown AGN contribution to SCUBA galaxy fluxes.

Further studies with more complete samples of GRB hosts will overcome some of the selection effects discussed above. If the discrepancy between the model and the observations continues to increase in significance then models of SCUBA galaxy evolution will need to include the implications of this result.

\section{REFERENCES}

1. Sanders, D. B., and Mirabel, I. F., $A R A \& A$, 34, 749-+ (1996).

2. Holland, W. S., Robson, E. I., Gear, W. K., and et al., MNRAS, 303, 659-672 (1999).

3. Smail, I., Ivison, R. J., Blain, A. W., and Kneib, J.-P., MNRAS, 331, 495-520 (2002).

4. Frayer, D. T., Ivison, R. J., Scoville, N. Z., Yun, M., Evans, A. S., Smail, I., Blain, A. W., and Kneib, J.-P., ApJL, 506, L7-L10 (1998).

5. Frayer, D. T., Ivison, R. J., Scoville, N. Z., Evans, A. S., Yun, M. S., Smail, I., Barger, A. J., Blain, A. W., and Kneib, J.-P., ApJL, 514, L13-L16 (1999).

6. Neri, R., Genzel, R., Ivison, R. J., Bertoldi, F., Blain, A. W., Chapman, S. C., Cox, P., Greve, T. R., Omont, A., and Frayer, D. T., ApJL, 597, L113-L116 (2003).

7. Chapman, S. C., Blain, A. W., Ivison, R. J., and Smail, I. R., Nat., 422, 695-698 (2003).

8. Chapman, S. C., Windhorst, R., Odewahn, S., Yan, H., and Conselice, C., astro-ph/0308197 (2003).

9. Blain, A. W., Smail, I., Ivison, R. J., Kneib, J.-P., and Frayer, D. T., Phys. Rep., 369, 111-176 (2002).

10. Almaini, O., Scott, S. E., Dunlop, J. S., Manners, J. C., Willott, C. J., Lawrence, A., Ivison, R. J., Johnson, O., Blain, A. W., Peacock, J. A., Oliver, S. J., Fox, M. J., Mann, R. G., Pérez-Fournon, I., González-Solares, E., Rowan-Robinson, M., Serjeant, S., Cabrera-Guerra, F., and Hughes, D. H., MNRAS, 338, 303-311 (2003).

11. Lilly, S. J., Eales, S. A., Gear, W. K. P., Hammer, F., Le Fèvre, O., Crampton, D., Bond, J. R., and Dunne, L., ApJ, 518, 641-655 (1999).

12. Archibald, E. N., Dunlop, J. S., Jimenez, R., Friaça, A. C. S., McLure, R. J., and Hughes, D. H., MNRAS, 336, 353-362 (2002).

13. Granato, G. L., DeZotti, G., Silva, L., Danese, L., and Magliocchetti, M., ApSS, 281, 497-500 (2002).

14. Hjorth, J., Sollerman, J., Møller, P., Fynbo, J. P. U., Woosley, S. E., Kouveliotou, C., Tanvir, N. R., Greiner, J., Andersen, M. I., Castro-Tirado, A. J., Castro Cerón, J. M., Fruchter, A. S., Gorosabel, 
J., Jakobsson, P., Kaper, L., Klose, S., Masetti, N., Pedersen, H., Pedersen, K., Pian, E., Palazzi, E., Rhoads, J. E., Rol, E., van den Heuvel, E. P. J., Vreeswijk, P. M., Watson, D., and Wijers, R. A. M. J., Nat., 423, 847-850 (2003).

15. Stanek, K. Z., Matheson, T., Garnavich, P. M., Martini, P., Berlind, P., Caldwell, N., Challis, P., Brown, W. R., Schild, R., Krisciunas, K., Calkins, M. L., Lee, J. C., Hathi, N., Jansen, R. A., Windhorst, R., Echevarria, L., Eisenstein, D. J., Pindor, B., Olszewski, E. W., Harding, P., Holland, S. T., and Bersier, D., ApJL, 591, L17-L20 (2003).

16. Waxman, E., and Draine, B. T., ApJ, 537, 796-802 (2000).

17. Barnard, V. E., Blain, A. W., Tanvir, N. R., Natarajan, P., Smith, I. A., Wijers, R. A. M. J., Kouveliotou, C., Rol, E., Tilanus, R. P. J., and Vreeswijk, P., MNRAS, 338, 1-6 (2003).

18. Watson, D., Reeves, J. N., Osborne, J., O’Brien, P. T., Pounds, K. A., Tedds, J. A., Santos-Lleó, M., and Ehle, M., A\&A, 393, L1-L5 (2002).

19. Berger, E., Cowie, L. L., Kulkarni, S. R., Frail, D. A., Aussel, H., and Barger, A. J., ApJ, 588, 99-112 (2003).

20. Ramirez-Ruiz, E., Trentham, N., and Blain, A. W., MNRAS, 329, 465-474 (2002).

21. Frail, D. A., Bertoldi, F., Moriarty-Schieven, G. H., Berger, E., Price, P. A., Bloom, J. S., Sari, R., Kulkarni, S. R., Gerardy, C. L., Reichart, D. E., Djorgovski, S. G., Galama, T. J., Harrison, F. A., Walter, F., Shepherd, D. S., Halpern, J., Peck, A. B., Menten, K. M., Yost, S. A., and Fox, D. W., ApJ, 565, 829-835 (2002).

22. MacFadyen, A. I., and Woosley, S. E., ApJ, 524, 262-289 (1999).

23. Vreeswijk, P. M., Moller, P., and Fynbo, J. P. U., astro-ph/0308164 (2003). 